A puntes del CENES

ISSN 0120-3053

Vol. XXIX - №. 50

Págs. $9-26$

II Semestre de 2010

\title{
Economía y medio ambiente
}

Ciro Alfonso Serna M endoza*

Fecha de recepción: 18 de agosto de 2010

Fecha de aprobación: 30 de septiembre de 2010

Clasificación JEL: E10, D62, R13

\footnotetext{
* Docente investigador de la Facultad de Economía, Universidad de Manizales, Colombia. Correo electrónico: redesomciro@hotmail.com
} 


\section{Resumen}

En ciertos escenarios académicos, políticos, sociales y ambientales se declara que el modelo económico dominante o los conceptos que lo integran son responsables de que el bienestar económico implique malestar ecológico.

Al partir de este supuesto, una forma de comprender las causas y de contribuir a la solución de la problemática ambiental es develar en qué consisten, en qué términos plantean la relación hombre-medio ambiente, las propuestas que incluyen la dimensión ambiental en el campo de la economía. Y si, al igual que las teorías ambientales, acuden a la ética como factor adecuado para disminuir las externalidades ${ }^{1}$ negativas generadas en el ambiente por la actividad económica.

Palabras clave: modelo económico, externalidades, bienestar económico.

\section{Abstract}

In certain academic, political, social, and environmental scenes it is declared that the dominant economic model or the concepts that integrate it are responsible for which the economic well-being implies ecological malaise. Starting off of this assumption, a form to include/ understand the causes and to contribute to the problematic solution of the environmental oneis to reveal in what they consist, in what terms raise the relation man-environment, the proposals that include the environmental dimension in the field of the economy, and if like the environmental theories, go to the ethics like suitable factor to diminish the negative externalities generated in the atmosphere by the economic activity.

Key words: economic model, externalidades, economic well-being.

\footnotetext{
La externalidad es considerada como la situación en la que un agente genera un nivel positivo (beneficio externo, externalidad positiva o economía externa) o negativo (costo externo, externalidad negativa o des-economía externa) de bienestar a un tercero. Un costo externo existe cuando un agente provoca una pérdida de bienestar a otro agente, y no la compensa. Cuando existe compensación por parte del agente que causa la externalidad, se dice que el efecto se internaliza. PEARCE, David y TURNER, Ferry (1995), "Economía de los recursos naturales y del medio ambiente, Colegio de Economistas de Madrid, Celeste Ediciones, 93-94, Madrid.
} 
"La E conomía moderna se ha visto sustancialmente empobrecida por la distancia creciente entre economía y ética".

Amartya Sen

\section{Introducción}

Al interpretar las discusiones actuales relacionadas con la crisis ambiental se devela que es frecuente sugerir la racionalidad económica como causa principal de esta situación, pero es poco habitual encontrar discursos estructurados al respecto, lo cual implica determinar en qué consiste la racionalidad económica y cuáles son los planteamientos determinantes en la relación hombreambiente que la hacen responsable del problema ambiental.

La teoría económica tiene su fundamento en los argumentos de A dam Smith sobre el equilibrio natural de las motivaciones, la cual sostiene que la conducta humana es integrada y movida por seis aspectos: egoísmo, conmiseración, deseo de ser libre, sentido de propiedad, hábito del trabajo y tendencia a permutar y cambiar una cosa por otra, factores que representan a su vez los intereses del hombre. En este sentido la libertad humana es fundamental para que cada hombre satisfaga sus intereses y se realice de forma natural. Cada sujeto, al buscar su propio beneficio, es conducido por una mano invisiblea promover un fin, el bien común, aunque este no haga parte de su propósito. De esas premisas Smith deduce que todo hombre es capaz de comportarse de una manera racional, o sea, de obtener los beneficios máximos con los recursos dados, o de reducir al mínimo sus costos al alcanzar un nivel dado de aprovechamiento particular y colectivo ${ }^{2-3}$; a este criterio se le denomina racionalidad

GODELIER, Maurice, (1979), "Racionalidad e Irracionalidad en Economía", Siglo Veintiuno Editores, México.

3 WEBER, Max, (2004), "Economía y Sociedad", Fondo de Cultura Económica, Madrid. 
económica o principio de maximización de utilidad

U na economía que busca esencialmente utilidad tiene como fundamento una teoría subjetiva del valor, por lo cual el comportamiento racional en las actividades de los agentes que toman decisiones es insuficiente para asegurar la racionalidad general del sistema, porque este tiende a la acumulación de capital y al desarrollo ilimitado de la fuerza productiva y no a la satisfacción de las necesidades de la sociedad, lo que ocasiona desequilibrio entre las capacidades de producción y el consumo de la sociedad, y entre las condiciones de producción de utilidad y las de su realización.

L a gran depresión, la desigualdad N orteSur, la apertura de la brecha entre ricos y pobres, la crisis ambiental, son algunas de las situaciones que reflejan las irregularidades producto de la racionalidad económica a nivel global; lo realmente extraño es que, aun conociendo estas limitaciones, la ciencia económica sustente su estructura teórica sobre la equivalencia entre la racional idad económica y la racional idad del ser humano a pesar de las continuas reflexiones que le exigen otros campos del saber. A fortunadamente a partir de los planteamientos expuestos por $\mathrm{G}$. A . A kerlof, A. M. Spence y J. E. Stiglitz, en su análisis de los mercados con información asimétrica, y los de Daniel $K$ ahneman sobre la racionalidad (o irracionalidad) de los seres humanos en función de la información de que disponen, trabajos reconocidos con el Premio N obel de E conomía en los años 2001 y 2002, respectivamente, la ciencia económica, aunque no todos los economistas y agentes económicos, replantea su concepción de racionalidad al incluir al gunos elementos que explican por qué son posibles diferentes sistemas de medición de costos y estimaciones de valores en un mismo individuo, y por qué ciertos comportamientos no pueden ser explicados con un único sistema de racionalidad, el económico.

Para analizar por qué la racionalidad económica puede ser un factor determinante en la crisis ambiental mundial, vale la pena precisar que la ciencia económica contemporánea entiende por racionalidad un sistema de ordenación de preferencias, o una forma de elegir, que está relacionado con el número de alternativas de elección y determinado por la diversidad de criterios, principios o razones adoptados por una persona de acuerdo con la época, la cultura y la sociedad, que le sirven para ordenar sus opciones en el momento de tomar la decisión. Por tanto, cada al ternativa tiene un valor y un costo que son variables y que dependen de los criterios de medición construidos a partir del conocimiento que posee cada ser humano.

El concepto de sistemas de racionalidades es fundamental para la economía, en cuanto define que el hombre tiene varias racionalidades 
(instintiva, tradicional, política y financiera) y que aunque algunas veces coincidan por la existencia de un conocimiento perfecto sobre una realidad, hay ocasiones en las cuales la coherencia (concordancia) no es posible entre ellas y una prevalecerá sobre las otras al momento de decidir. Lo que hacen las racional idades es establecer el orden de preferencia entre las alternativas, en relación con las estimaciones de costes y beneficios establecidas de acuerdo con la información disponible; por lo que la racionalidad o irracionalidad depende del nivel de asimetría de información coherencia y calidad de esta 0 de la capacidad humana para evaluar las informaciones de que dispone.

Daniel Kahneman y Tversky han demostrado que si un ser humano tiene que elegir entre dos posibilidades, A y $B$, elegirá una de estas dos opciones, pero si se le ofrecen alternativas "triviales", modificará el orden de preferencias entre A y $B$; es decir, su elección podría ser diferente a la inicial. Estas posturas, aparentemente irracionales, son frecuentes y observables en las situaciones calificadas en la teoría de juegos como dilema del prisionero. Al el egir entre dos situaciones alternativas, "todos cooperan" y "ninguno coopera"; es muy probable que todos elijan la primera alternativa, porque el valor para todos es mayor al de la segunda; si se adicionan otras dos posibilidades, "yo coopero y los demás no" y "los demás cooperan y yo no", entonces la decisión racional de todos los agentes es diferente y probablemente pone en riesgo la cooperación.

El dilema del prisionero y otros problemas de racionalidad colectiva en los que los objetivos planteados por seres racionales no pueden alcanzarse teóricamente de una forma óptima permiten reflexionar sobre cuáles son las posibilidades de maximizar el bienestar social, si sólo se persiguen fines que maximicen el bienestar individual. Este es un asunto de relevancia práctica para la teoría económica cuando se pretenden conductas cooperativas encaminadas a superar la injusticia intergeneracional que enfrenta hoy la humanidad en lo relacionado con el medio ambiental.

En cuanto a la utilización, recuperación y cuidado del medio ambiente, existen diversas alternativas: todos cooperan, ninguno coopera, yo coopero y los demás no y los demás cooperan y yo no. Un país como Estados Unidos, que aporta aproximadamente el $30 \%$ de la contaminación mundial, decide no cooperar, negándose a ratificar el Protocolo de K yoto, lo que genera nuevas opciones para otros agentes al momento de decidir, porque conocerán diferentes alternativas y los costos-beneficios generados en que han incurrido otros agentes de acuerdo con la elección de una alternativa específica; esta información será estimada según las diversas racionalidades. Si el medio ambiente es un recurso más por optimizar y no un sistema complejo de relaciones que tiene efectos directos sobre los seres vivos y 
las actividades humanas en el tiempo, las actitudes que se dan ante éste serán variadas, porque estarán determinadas por las concepciones e información que tenga cada individuo; por tanto, la probabilidad de que existan actos irracionales frente al medio ambiente será mayor si la información al respecto es insuficiente o parcial.

K ahneman y T versky en 1979 señalaron otro problema adicional que debe superar la economía al imponer la utilidad como criterio predominante de decisión: el efecto certidumbre 0 tendencia a subestimar los resultados que son sólo probables en comparación con los resultados que son obtenidos con seguridad. Esta dificultad de hacer estimaciones en situaciones de riesgo 0 de información insuficiente sobre las consecuencias de una elección favorece la aversión al riesgo en elecciones que implican ganancias seguras y la preferencia por el riesgo en elecciones que implican pérdidas seguras. En cualquier caso los eventos en el largo plazo son siempre menos seguros y documentados que los sucesos del corto plazo; en el caso del medio ambiente esta tendencia explica de al guna manera la resistencia a ejecutar en el presente las acciones propuestas para superar la dificultad ambiental de una forma eficiente, dado que cuanto mayor sea el periodo de cuestión para que ocurra una situación, el valor estimado sobre sus costos y beneficios será menor.

G. A. A kerlof, A. M. Spence y J. E.
Stiglitz, en sus análisis de los mercados con información asimétrica, develan que la desigual dad en la información no sólo es una limitante de la racionalidad económica por su impacto en la asignación eficaz de los recursos, sino que es un inconveniente común a todas las formas de racionalidad. L a economía ortodoxa construye su concepto de racionalidad de mercado sobre el supuesto de que todos los agentes económicos tienen acceso sin ningún costo, a toda la información relevante para sus decisiones. Situación que día a día está más lejana de la realidad; en general se percibe que una de las partes que interviene en una transacción económica tiene más información que la otra, ventaja que tiene un costo que usualmente es elevado.

Cuando se habla de la superación de los límites de la resiliencia planetaria, o del desequilibrio ambiental ocasionado por una demanda social mayor a la oferta natural del sistema, se advierte que los productores tienen una información completa de los desechos que debe absorber el sistema desde el momento de la producción hasta el del consumo del producto, información que el consumidor desconoce o conoce parcialmente; éste probablemente tomaría otra opción si tuviera la certeza de que por consumir el bien tendrá que pagar un costo adicional por la contaminación que está ocasionando, o de que está asumiendo un costo que el productor conoció, pudo evitarlo o asumirlo, pero se lo trasladó. Es el caso de los empaques que no son 
biodegradables, en el que el productor disminuye sus costos al colocar su producto en el mercado a un precio inferior al de otro productor de la industria, que utiliza una metodología ecológicamente eficiente para desarrollar su actividad, modificando el precio del mercado del bien a un nivel que no es óptimo.

\section{Relación entre ética, medio ambiente y economía}

Es necesario un análisis de las concepciones teóricas acerca de la relación hombre-ambiente, las cuales hacen parte de un sistema de valores y de racional idad contenidos en la ciencia económica, y las implicaciones de adoptar dichos significados en la construcción e interpretación de la realidad y, por consiguiente, en el comportamiento humano ante el medio ambiente, con el fin de develar si esta visión del mundo es en realidad responsable de que las acciones humanas sean irracionales frente al medio ambiente.

En la literatura económica usualmente se describe al hombre como un ser individualista y autónomo que sólo piensa en maximizar sus propios intereses, casi siempre definidos en términos monetarios. En la misma perspectiva el concepto de medio ambiente corresponde a la idea de naturaleza, reducida a la categoría de cosa dominada que puede ser infinitamente dominada, la cual es considerada como un elemento externo del sistema económico y cuyo valor es determinado en el mercado; el resultado es una cosmovisión que reduce la sociedad a un conjunto de recursos humanos y la naturaleza a una suma de recursos naturales.

A doptar estos conceptos económicos como criterios básicos para entender la existencia humana exige de al gún modo circunscribir la finalidad del ser humano a una vida llena de riqueza material y el medio ambiente a un conjunto de recursos naturales para explotar.

L a ciencia económica, sustentada en una concepción del hombre como individuo racional y egoísta, parte de una idea de ser humano dominado por sus preferencias, incapaz de actuar sobre la base de las restricciones a la maximización de su satisfacción 0 utilidad; esta simplificación promueve el individualismo, universaliza el valor monetario o de cambio como unidad de medida aplicable a la total idad del mundo físico y sociocultural, reduce el concepto de bienes a los que son mercantiles, los cuales pueden ser monetizados, limita las reflexiones éticas al marco hedonista y utilitarista, porque la finalidad del ser humano se limita a satisfacer las necesidades y maximizar la utilidad, aceptando de alguna forma cualquier medio, ya que la norma básica de comportamiento es lograr un mayor beneficio.

Este punto muestra lo relativo que es el concepto de comportamiento económico 
racional, respecto a los individuos que toman decisiones, puesto que no se tiene la responsabilidad de evaluar las consecuencias de estas, dada la existencia del mercado planteado como mecanismo que ejerce control y provee equilibrio a nivel general ${ }^{4}$.

C abe cuestionarse cómo una entidad que no asume la función de evaluar la bondad o maldad de las situaciones que se den en su interior puede ser justa y cómo el hombre renuncia a ser sujeto moral sobre los actos económicos y delega para este fin al mercado, sabiendo que debe hacer reflexiones éticas acerca de cualquier acto que él realice con su capacidad de conocimiento y decisión. Pero esta labor no sólo concierne a los economistas, sino que ha de exigirse en todos los actores de la vida social, toda vez que los mercados no existen de manera natural ni actúan de forma invisible, pues son organizados y construidos tanto social como políticamente. AI tener claro esto la humanidad demanda un sistema de desarrollo social económicamente sostenible, que supere los problemas generados en el medio ambiente global, con los que ha comprometido la calidad de vida de los seres vivos actuales y futuros.

A Igunas concepciones económicas que introducen el tema del medio ambiente plantean que este, como todo objeto sobre el cual no existe una propiedad definida y un conocimiento acerca de su valor real, tiende a ser abusado por la sociedad y los agentes económicos; a esta situación se le conoce como "la tragedia de los bienes comunes". Si se conociera el precio o se estableciera un costo por el uso de los recursos naturales que no fuera inferior al que corresponde, las actuaciones humanas serían diferentes. Esto explica los múltiples intentos de los economistas por tratar de asignar un precio adecuado para dejar actuar al mercado y la convicción de que la degradación ambiental producida asegure la supervivencia de todos los seres y la biosfera, es decir, que exista un nivel de contaminación óptimo que maximice el bienestar de la sociedad por tener en cuenta sus recursos, preferencias y parámetros económicos.

Para cumplir este objetivo se estudia la forma de explotación de los recursos naturales apropiados o apropiables individualmente, con el fin de buscar al ternativas que permitan la incorporación de las externalidades en el costo o precio del producto, por medio de incentivos económicos como las tasas óptimas de extracción de recursos que una vez regulado el precio, se plantea en términos de condiciones de oferta, demanda, tasa de interés y tasa de descuento, pero que se calcula independientemente de la tasa de reposición natural. Sin embargo, es de anotar que muchas fórmulas demuestran la conveniencia de agotar

4 SUDOW SKI, Zdzislaw, (1983), "Conceptos teóricos de la racionalidad económica”. Fondo de Cultura Económica, 5859, México. 
hasta su extinción un recurso natural de lenta reposición, ignorando los límites del ambiente como fuente y destino de los residuos de los procesos de producción y consumo, establecido en el principio universal de la conservación de la materia. Se propone sustituir el problema de la contaminación por el factor de distribución de los costos de contaminación, dejando de lado preguntas como por qué y cómo se contamina y reemplazándolas por cómo convertir la contaminación en una mercancía más, sujeta al juego de oferta y demanda del mercado 5 .

Encontrar un valor para el ambiente debe pasar de ser un ejercicio matemático a un proceso de construcción social dinámico que incluya las variaciones generadas por la percepción y valoración del ambiente, según el momento histórico y la sociedad de referencia y el valor intrínseco que existe en esta entidad, que usualmente es omitido y que posee independientemente de las apreciaciones humanas. Entender cómo se da el proceso de relación entre el hombre y su medio ambiente y cómo se puede influir en este puede ser más eficiente que diseñar métodos para asignarle un valor monetario que probablemente sea inadecuado.

Al partir de que los valores económicos son entidades que reflejan las preferencias de las personas, la ciencia económica ha tratado de evitar errores en la valoración del ambiente, creando una serie de conceptos con relación al val or como valor de uso, val or de uso por individuos futuros, valor de uso por otros, valor actual, valor de opción, valor intrínseco o de existencia, valores legados, entre otros, que tienden a buscar los valores económicos totales para tratar de superar la irreversibilidad 0 probabilidad de eliminación de un activo con poca o ninguna posibilidad de regeneración, la incertidumbre 0 desconocimiento del futuro y la singularidad, entendida como el valor que reside en algo y que no está relacionado en absoluto con los seres humanos, característica denominada también valor intrínseco o de existencia, que da lugar al vínculo entre los ecologistas y los economistas con al gún tipo de al truismo 0 preocupación por otras personas u otros seres vivos, lo cual se expresa en las motivaciones de dejar un legado, de hacer una donación o de ser consistente con la simpatía por las personas 0 animales.

Se encuentran argumentos que sugieren que el altruismo también puede incluirse en el model o de racional idad económica clásica, porque de una u otra forma se tiende a maximizar la utilidad o el bienestar, esta vez en el donante. De ser así cabe preguntarse cuáles pueden ser otros motivos relevantes, diferentes al altruismo, para explicar el valor de existencia del medio ambiente. Los motivos que comúnmente se han expuesto para valorar la existencia del medio ambiente son los derechos que tienen los seres no humanos, concepción

5 GUTMAN, Pablo, (1994), “Ciencias sociales y formación ambiental”, Gedisa, 125 -155, Barcelona 
identificable en las ideologías biologistas, zoo y biocéntricas, y el motivo $G$ aia basado en la idea de que la Tierra es un organismo vivo que se ajusta de un modo autorregulador a las agresiones externas, postura conocida como ecocentrismo o gaicentrismo, en la que los humanos son unos individuos más de la biosfera y por tanto no son importantes como agentes de control, preservación o conservación de la misma.

Sin negar que existe este tipo de motivaciones, es importante recalcar que estos planteamientos promueven una preservación extrema del medio ambiente, lo que implica necesariamente, un cambio radical para la humanidad, la cual debe reducir sus actividades a la supervivencia, es decir, estos enfoques reducen el hombre a su biología.

Cuando se analizan los indicadores ambientales se observa que los avances en la recuperación del equilibrio ambiental no son tan alentadores como se esperaba, Io cual permite inferir que el comportamiento de todos los hombres no es racional y, mucho menos, que este contribuye a un fin común como lo planteaba Smith, que en este caso estaría representado en un medio ambiente óptimo para el desarrollo de todas las actividades humanas. Esto muestra lo relativo que es el concepto de comportamiento económico racional, respecto a los que toman las decisiones que comprometen los recursos naturales, porque a eso reducen su medio ambiente. Esto quiere decir que se confunde una racionalidad relativa, limitada por los conocimientos de los que toman decisiones, con la racionalidad absoluta de la asignación óptima cuando existe un conocimiento perfecto.

La limitación y deformación de la noción de racionalidad en la economía se reflejan en que se ha permitido una congregación de términos que se usan indistintamente para referirse a lo que es racional, como la eficacia, eficiencia, rentabilidad, rendimiento, productividad, minimización de costos, utilidad máxima, entre otros ${ }^{6}$.

Pero por qué preocuparse por la racionalidad; al respecto la literatura que plantea este tema sostiene que el concepto de racionalidad es aplicable a tres dimensiones fundamentales relacionadas entre sí: el pensamiento, la acción y los fines. Desde esta óptica este análisis de la racionalidad se refiere a dos tipos diferentes de razón, teórica y práctica, o dos tipos de facultades.

A sí que racionalizar la producción de ciertos bienes sin racionalizar la eliminación de los desechos que son consecuencia del proceso, o producir rupturas en el equilibrio del medio ambiente sin introducir racional mente nuevos equilibrios naturales, denota que el problema no es la racionalización,

6 SERNA MENDOZA, Ciro (2001), "El problema de la racionalidad económica desde la perspectiva de godelier", Departamento de publicaciones Universidad de Manizales, 21-85, Manizales. 
teórica y práctica, sino más bien las racionalizaciones incompletas ocasio-nadas por criterios que, al ser inválidos siguen vigentes en la estructura teórica de los individuos y peor aun en la de las ciencias.

U na de las alternativas propuestas para evitar el deterioro ambiental que prueba que se confunde una racional idad relativa, limitada por los conocimientos de los que toman decisiones, con la racionalidad absoluta de la asignación óptima del conocimiento perfecto -que no es real- , es la interiorización de externalidades, aumentando el costo de los bienes, y por lo tanto su precio de mercado, para reducir su consumo. EI resul tado de este proceso es que sólo los individuos con recursos monetarios suficientes pueden disfrutar del bien y de un mejor y mayor acceso a los recursos naturales, puesto que tienen con que pagar por eso, y la degradación del medio natural conllevaría una decadencia del medio social, porque esta alternativa fomenta desigualdades intergeneracionales que deben afrontar los seres vivos en el tiempo, así sean parcialmente superadas en el corto plazo ${ }^{7}$.

El comportamiento económico racional puede producir resultados considerados irracionales desde la perspectiva colectiva 0 de la sociedad en conjunto, lo cual crea tensiones socioeconómicas que originan desequilibrio en el sistema y dificulta la posibilidad de asumir un comportamiento racional en todas las actividades y dimensiones humanas ${ }^{8}$ que deben ser controlados por el Estado, que a través del intervencionismo trata de armonizar los desajustes a que da origen el modo de producción capital ista fundamentado en la racionalidad económica. Estos resultados dependen de la naturaleza y del número de los sectores donde se encuentra bloqueado el proceso de realización del valor y de la utilidad ${ }^{9}$.

En general las decisiones adoptadas con la racionalidad financiera y la instintiva tenderán a valorar más el corto que el largo plazo; sin embargo, las racionalidades tradicional y política parecen tener una forma de ponderación que premia los resultados a largo plazo.

A Igunos autores afirman que etimológicamente se puede establecer una relación entre Economía y Ecología, por ser términos que provienen del griego oikos, que significa casa. Para ellos la E conomía en un principio significaba sólo la administración de la casa, de los bienes familiares, luego se amplió su significado a la administración de todos lo bienes y a las leyes que regulan el mercado de los valores monetarios, y la Ecología es definida como el conjunto de conocimientos referentes a la economía de la natural eza, que observa la lógica con la cual esta administra sus bienes, es decir, todos los recursos naturales. De esta

\footnotetext{
7 GONZÁlEZ ÁlVAREZ, Luis (1993), "Ética Ecológica para América Latina”, Códice, 75-79, Bogotá.

8 SERNA MENDOZA, Ciro (2001), "El problema de la racionalidad económica desde la perspectiva de Godelier", Departamento de Publicaciones Universidad de Manizales, 21-85. Manizales.

9 GODELIER, Maurice (1979), "Racionalidad e irracionalidad en economía", Siglo Veintiuno, 68, México.
} 
perspectiva se puede decir que aunque estas ciencias estaban íntimamente ligadas en sus orígenes, con el tiempo tomaron caminos divergentes, pero que a raíz de la complejidad ambiental el tema de la natural eza exige integrar teóricamente los conceptos desarrollados sobre el medio ambiente, en estos y otros campos del saber, porque a partir de estos criterios el hombre consolida su sistema racional y establece su forma de relacionarse consigo mismo y con todo lo que le rodea.

L os enfoques económicos acerca del medio ambiente están unidos con los ecológicos, en cuanto este es usualmente reducido a natural eza o recursos naturales como lo considera la Economía A mbiental; no obstante, la Economía Ecológica y el desarrollo sostenible plantean ideas amplias que están contenidas en el siguiente concepto: el medio ambiente es un sistema complejo de relaciones físicas, químicas, biológicas, sociales, políticas, económicas y culturales, con gran sensibilidad a las variaciones de cada uno de sus componentes, que producen efectos, directos o indirectos, sobre los seres vivos y las actividades humanas a corto, mediano o largo plazo.

L a economía hace al gunas propuestas que tienen el objetivo de compatibilizar la protección del ecosistema y el crecimiento económico, para que este sea posible en el largo plazo; en este caso se habla de ideologías tecnocéntricas y ecocéntricas.
La ideología tecnocéntrica contiene dos visiones: extrema comucopía, posición de explotación de recursos orientada hacia el crecimiento y reflejada en la Economía A mbiental, queotorga un val or instrumental a la naturaleza, porque el hombre es considerado lo más importante del mundo (esta idea se ha catalogado como antropocentrismo fuerte) y la acomodativa, perspectiva conservadora y de gestión de los recursos, identificable en la ideología del desarrollo sostenible, que aunque tiene una visión integral sigue otorgándole valor instrumental al medio ambiente; en esta posición la centralidad del hombre parece que no supera el tratamiento despótico de este frente a la natural eza.

EI enfoque de ecología profunda incluye a su vez la perspectiva comunalista, que sostiene una posición de preservación de los recursos naturales, como lo sugiere la Economía Ecológica, y otorga un valor instrumental e intrínseco a la naturaleza; en este caso la centralidad del hombre no impide que este se responsabilice de todos los actos; esto es lo que al gunos denominan antropocentrismo débil. La ideología ecocéntrica, que asume una posición de preservación extrema justificada en los derechos morales 0 intereses que poseen las entidades no humanas, plantea un sistema socioeconómico con un gasto mínimo de recursos naturales, al sugerir alternativas como la agricul tura orgánica y la desindustrialización ${ }^{10}$. 10 O'RIORDAN, Tim. y TURNER, Ferry, (1983), "An Annotated Reader in Environmental Planning and Management”,
Pergamon Press, Oxford. 
Según el análisis marxista, los sistemas naturales pueden suponer límites a la capacidad de reproducción del sistema productivo, así como a la consistencia económica y política de la sociedad. EI balance de materiales a largo plazo cuestiona si el cambio tecnológico alivia o agrava las presiones que el medio ambiente impone a la posibilidad de reproducirse y si lo hace coherente con el sistema social para que sea razonablemente estable ${ }^{11}$.

Los "institucionalistas" aceptan Ios costos sociales de la contaminación e insisten en la importancia de los cimientos ecológicos de cualquier sistema económico. Los argumentos anti-crecimiento fueron reforzados por análisis económicos que destacaron los costos sociales, especialmente los costos ambientales de vivir en una sociedad en crecimiento; entre éstos están la Paradoja de Easterlin, que expone que no hay correlación estrecha entre la abundancia material y la felicidad humana; el concepto de los Bienes de Posición de Hirsch, que propone que el disfrute de una serie de bienes está necesariamente limitado a un pequeño grupo de grandes ingresos aunque exista la posibilidad de consumo en todos los segmentos sociales; y el A nálisis de la Economía Triste de Scitovsky, en la que las necesidades humanas van más allá de la afluencia material. Estas ideas son representativas del pensamiento de los límites sociales del problema.

Las consideraciones más frecuentes sobre la crisis ambiental apuntan a que esta puede definirse como una falla con raíces en la actividad económica con una base ética insuficiente, centrada en las relaciones entre personas y entre estas y la sociedad, en la que habitualmente las interacciones hombre natural eza son omitidas o establecidas de acuerdo con lineamientos económicos utilitarios, en los cuales establecen derechos pero no obligaciones con el hábitat.

A I respecto L eopold propone investigar cada cuestión en términos de lo que es ética y estéticamente correcto, así como ventajoso económicamente, al tener en cuenta que algo es correcto cuando tiende a conservar la integridad, la estabilidad y la belleza de la comunidad biótica, e incorrecto cuando tiende a todo lo contrario, y renunciar a la idea de que el uso adecuado de la tierra es sólo un problema económico; añade que la eficiencia económica no puede elegirse como único criterio de decisión económica. Se trata de integrar los conceptos ambientales y económicos a la perspectiva ética, con el fin de tener una evidencia suficiente al momento de elegir, que permita argumentar acerca de la justicia de incluir a toda la humanidad -presente y futura- en las decisiones, y así responder a cuestiones ecológicas y sociales sin entrar en total

11 PEARCE, David y TURNER, Ferry, (1995), "Economía de los recursos naturales y del medio ambiente, Colegio de Economistas de Madrid, Celeste Ediciones, 35, 42-43, Madrid. 
contradicción con los sistemas de racionalidad existentes ${ }^{12}$.

La relación entre Economía y Ética se hace co-dependiente en este nivel, porque el objetivo material de estas ciencias es el actuar humano, entendido como acto voluntario, inteligente y libre; la diferencia radica en su objetivo formal, la Economía investiga el comportamiento humano relacionado con la asignación de medios escasos y de uso alternativo para la consecución de fines, y el objetivo formal dela Ética son los actos humanos, que clasifica como buenos o malos, según su ordenabilidad al fin o bien de cada ser humano, en el contexto del respeto a la naturaleza.

$M$ arx plantea que existe una identidad entre el hombre y la naturaleza, ya que el hombre se objetiviza en la naturaleza, es decir, es en ella donde el hombre se encuentra y se perfecciona, pues ésta le proporciona el espacio de acción, el instrumento, y porque también él es natural. La arrogancia del hombre convierte la natural eza en un instrumento útil para el desarrollo evolutivo de la humanidad, sin tener en cuenta la existencia de otras especies en la tierra, irrespetando, por tanto, los espacios de cada una de las especies e inter rumpiendo las funciones de cada ecosistema.
L udwing V on M ises, Gabriel Zanotti y M urray Rothbard exponen que la E conomía no limita su campo de acción al aspecto material, porque lo económico no equivale a lo material, al exponer que el objeto formal de la Economía es la deducción de las implicaciones lógicas de la acción humana, ya que no hay diferencia entre las acciones que se traducen en precios monetarios y las que se traducen en precios no monetarios ${ }^{13}$.

L os actos humanos son los que proceden de la libertad, por lo tanto en ellos se considera la finalidad material 0 espiritual de la acción, en este punto se puede afirmar que "la esfera de la economía es más grande de lo que tradicionalmente ha sido definido por los economistas"; toda decisión económica es un acto humano, esto quiere decir que se puede entender la ciencia económica cuando se tiene un enfoque centrado en la comprensión de la natural eza del acto humano ${ }^{14}$. La E conomía es un producto humano y no abarca toda la acción humana, porque el hombre no se agota en su acción económica.

A unque la función de la Economía no es la formulación de juicios, es relevante señalar que el pensamiento económico sólo se da en la mente de los humanos, únicos seres conocidos capaces de valorar y juzgar moralmente. En

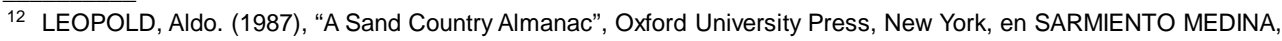
Pedro José, "Bioética y medio ambiente. Introducción a la problemática bioético-ambiental y sus perspectivas", en Revista Persona y Bioética, año 5, No. 13-14, Universidad de La Sabana, 20- 23, Bogotá.

13 MURRAY, Rothbard. (1980), "Individualism and the Philosophy of the Social Sciences", Cato Institute, 10 -37, San Francisco.

14 KIRZNER, Israel. (1976), "The economic point of view. Kansas City", Sheed and Ward, 184. 
consecuencia el acto del economista, o de un agente económico, como todo acto humano, es susceptible de ser juzgado moralmente; en palabras de Milton Friedman.

"Ios economistas no son sólo eso sino también seres humanos, y sus propios valores indudablemente afectan su economía (ciencia). Sin lugar a duda, los juicios de valor de un economista influyen en la selección de sus tópicos de análisis, y quizás también en sus conclusiones y estas a su vez afectaran sus juicios de valor. Pese a ello, esto no al tera el punto fundamental que, en principio ${ }^{15}$, no hay juicios de valor en economía".

Es indudable que existe una relación entre los juicios de val or de una persona y sus presunciones acerca de los hechos.

Al compartir el mismo objeto material desde diferentes perspectivas, el conocimiento puede ser óptimo si existe complementariedad entre Ética y Economía.

$L$ a relación entre las ciencias naturales (E cología) y sociales (E conomía) puede establecerse desde la Ética Ecológica, factor de unión entre estas dos áreas, que a su vez tiene soporte en la Ética
E conómica como factor de conexión con la realidad, es decir se debe incluir la Economía cuando se hace Ética Ecológica, estableciendo parámetros éticos y ambientales que contrasten el principio de maximización de utilidades como único criterio válido, sin sustituir la Ética Económica por la E cológica.

El reto que tiene la É tica es compatibilizar su proyección económica y ecológica, para que la producción, la distribución y el consumo de bienes sean establecidos por principios éticos compatibles y los criterios de elección sean más que económicos. La humanidad debe tener conciencia de que puede modificar la realidad de la naturaleza sin destruirla, de tal forma que exista armonía entre preservación y cuidado medioambiental con progreso y bienestar.

\section{Conclusiones}

Movimientos como el de Kansas City, Cambridge y el de E conomía Postautista ${ }^{16}$ estiman que el pensamiento económico delegó la administración recta y prudente de los bienes planteada por A ristóteles al mercado, al sustentar científicamente que este es un mecanismo social de regulación. Deal guna manera este deja de considerarse un instrumento al servicio de la humanidad y se transforma en un fin, sin importar las

\footnotetext{
15 FRIENDMAN, Milton. (1967), "Human Values and Economic Policy, A Symposium, Sydney Hook, New York University Press, 86-88, New York.

16 Propuesta de Kansas City, elaborada por investigadores, profesores y estudiantes de 22 países, el Movimiento de Cambridge, desarrollado en Reino Unido, y el Movimiento Económico Postautista, fundamentado en Francia, son algunas de las tendencias contemporáneas que plantean fallas de los enfoques educativos e investigativos actuales de la economía.
} 
incongruencias prácticas que se evidencian frecuentemente ante las que se acude a abstracciones que impiden confrontar las hipótesis establecidas, ocasionando un afianzamiento ciego a hipótesis en ausencia de datos 0 en presencia de datos adversos.

Es decir que el análisis económico está siendo fundamentado sobre fal acias, que a su vez son utilizadas para justificar políticas que afectan al bienestar de millones de seres humanos. Esta situación requiere definir enfoques educativos e investigativos en la Economía, que permitan analizar y explicar los problemas reales que afronta Ia humanidad, que incluyan ${ }^{17}$ :

Una concepción más amplia del comportamiento humano, que explique factores clave que influyen en la psicología económica de las personas, redefiniendo la concepción del "homus economicus".

Instituciones y sistemas de valores sociales, políticos, económicos y éticos que permitan o limiten la realización de elecciones particulares y creen identidades sociales o comunitarias determinantes en el comportamiento humano.

D esde la perspectiva histórica, análisis y estudios de procesos económicos para establecer cómo y por qué cambian las cosas de acuerdo con el espacio y el tiempo.
Integración entre lo positivo y lo normativo. Los valores del investigador se encuentran en la propia investigación científica y en las afirmaciones que surgen de la misma, y se evidencian en juicios más sofisticados y cercanos a la realidad, para que cumplan los lineamientos del rigor científico.

La medición experimental como instrumento indispensable para sustentar el realismo de las explicaciones teóricas que se realizan.

L a ampliación de los métodos de análisis como requisito para avanzar en la comprensión de los fenómenos económicos. Es preciso limitar la utilización exclusivay abusiva de modelos formal es y econométricos, dejando lugar para otros procedimientos como la observación, el análisis discursivo, los estudios de caso, etc., capaces de ampliar el estudio de los fenómenos desde diferentes perspectivas, mediante técnicas de asociación de las informaciones que puedan ofrecer nuevas y más completas percepciones de la real idad.

L a interdisciplinariedad para reconocer que existen diversas escuelas de pensamiento dentro de disciplinas y conocer los desarrollos alternativos en otras áreas afines, particularmente en el campo de las ciencias biológicas y sociales, que permiten un análisis de la

\footnotetext{
17 MAX-NEEF, Manfred, "La universidad y el desarrollo sostenible", en: Revista Asuntos Económicos y Administrativos, No 6, primer semestre, Facultad de Economía y Administración. Centro de Investigaciones Económicas, Universidad de Manizales, 17-21, Manizales. (ver también "La ciencia económica necesita una reforma fundamental y ahora es el momento del cambio").
} 
realidad en la cual se enmarcan los fenómenos económicos en su conjunto, lo que constituye, objetivamente, la esencia del quehacer intelectual.

Establecer nuevas estrategias para superar las actuales resistencias al cambio por parte de quienes ejercen el control sobre la disciplina en las diversas instituciones y la marginación de los economistas críticos. A Igunos agentes y economistas que se benefician en la situación actual se oponen al cambio, aunque reconocen que la Economía es una ciencia de la que la sociedad espera algo más que sofisticadas y elegantes abstracciones.

L a E conomía debe ser una disciplina útil para la humanidad y su medio ambiente, con una visión amplia e integral que permita combinar diversos enfoques y afrontar los problemas actuales de la humanidad, ocasionados por un despotismo entre lo humano y lo no humano, por lo cual la transdisciplinaridad es fundamental para establecer un modelo de desarrollo humano, caracterizado por la coherencia técnica, por coincidir con el verdadero sentido y razón de ser de la E conomía: "atender, con la mayor exigencia científica y ética la satisfacción de las necesidades de la sociedad" ${ }^{18}$.

L a E conomía debe resolver sus problemas desde lo humano y lo social, ampliando sus perspectivas de crecimiento hacia el bienestar, para lo cual requiere un mayor grado de abstracción y racionalidad científica, tal como lo intentan hacer corrientes como la economía sostenible, la ecológica, entre otras, que pretenden presentar propuestas óptimas y coherentes con los retos que afronta la humanidad en el siglo XXI, en cuanto a sus relaciones físicas, químicas, biológicas, sociales, políticas, económicas y culturales, es decir, con su medio ambiente y con las generaciones futuras.

\footnotetext{
$\overline{18}$ TORRES OSORIO, Pedro Antonio. (2004), "El poder de la Epistemología en la formación del economista”, en: Revista Asuntos Económicos y Administrativos, No 6, primer semestre, Facultad de Economía y Administración, Centro de Investigaciones Económicas, Universidad de Manizales, 67, Manizales.
} 


\section{Bibliografía}

FRIENDM AN, M ilton (1967), Human V alues and Economic Policy: A Symposium, Sydney Hook New Y ork University Press, 86-88, New Y ork.

GODELIER, M aurice (1979), Racionalidad e irracionalidad en Economía, Siglo Veintiuno Editores, M éxico.

GONZÁ LEZ, L uis J osé (1993), Ética E cológica para A mérica L atina, Códice L tda., 75-79, Bogotá.

GUTM AN, Pablo (1994), Ciencias Sociales y formación ambiental, Gedisa, 125-155, Barcelona.

KIRZNER, Israel (1976), The Economic Point of V iew, Sheed and Ward, 184, Kansas City.

LEOPOLD, Aldo (1987), A Sand Country Almanac, primera edición 1949, Oxford University Press, New Y ork, en SA RM IENTO, Pedro José, (2000), Bioética y medio ambiente, Introducción a la problemática bioético-ambiental y sus perspectivas, en Revista Persona y Bioética, año 5, N. ${ }^{\circ}$ 13-14, 20-23, Universidad de la Sabana, Bogotá.

M AX-NEEF, M anfred (2004), La universidad y el desarrollo sostenible, en Revista A suntos Económicos y Administrativos, N. ${ }^{\circ} 6$, primer semestre, 17-21, Facultad de Economía y Administración, Centro de Investigaciones Económicas, Universidad de Manizales, $M$ anizales.
M URRAY, Rothbard (1980), Individualism and the Philosophy of the Social Sciences. Cato Institute, $10-37$, San Francisco.

O'RIORDA N, Tim and TURNER, Ferry (1983), A $n$ A nnotated Reader in Environmental Planning and $\mathrm{M}$ anagement, Pergamon Press, Oxford.

PEARCE, David y TURNER, Ferry (1995), Economía de los recursos naturales y del medio ambiente, Colegio de Economistas de M adrid Celeste Ediciones, 42-43, M adrid.

SER N A, Ciro Alfonso (2001), El problema de la racionalidad económica desde la perspectiva de Godelier, Departamento de publicaciones, Universidad de $\mathrm{M}$ anizales, 21-85, M anizales.

SUDOWSKI, Zdzislaw (1983), Conceptos teóricos de la racionalidad económica, Fondo de Cultura Económica, 58 - 59, M éxico.

TORRES, Pedro A ntonio (2004), El poder de la E pistemología en la formación del economista, en Revista A suntos Económicos y Administrativos, N. ${ }^{\circ}$ 6, primer semestre, 67, F acultad de Economía y A dministración, Centro de Investigaciones Económicas, Universidad de Manizales, $M$ anizales.

WEBER, M ax (2004), Economía y sociedad, Fondo de Cultura E conómica, M adrid. 